\title{
Urine Alpha-1-Microglobulin Levels and Acute Kidney Injury, Mortality, and Cardiovascular Events following Cardiac Surgery
}

\author{
Jonathan G. Amatrudaa, b Michelle M. Estrella a, b, c Amit X. Garg d, e \\ Heather Thiessen-Philbrook ${ }^{f}$ Eric McArthure Steven G. Cocag \\ Chirag R. Parikh ${ }^{f}$ Michael G. Shlipak ${ }^{b, h}$ for the TRIBE-AKI Consortium \\ aDivision of Nephrology, Department of Medicine, University of California, San Francisco, San Francisco, CA, USA; \\ ${ }^{b}$ Kidney Health Research Collaborative, San Francisco VA Health Care System \& University of California, \\ San Francisco, San Francisco, CA, USA; 'Division of Nephrology, Department of Medicine, San Francisco VA Health \\ Care System, San Francisco, CA, USA; dSchulich School of Medicine and Dentistry, Western University, London, ON,

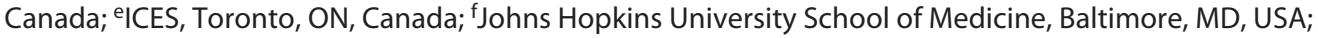 \\ IIcahn School of Medicine at Mount Sinai, New York, NY, USA; hDepartment of Medicine, San Francisco VA Health \\ Care System, San Francisco, CA, USA
}

\section{Keywords}

Acute kidney injury · Chronic kidney disease · Mortality ·

Biomarker · Alpha-1-microglobulin · Cardiac surgery

\begin{abstract}
Introduction: Urine alpha-1-microglobulin (Ua1m) elevations signal proximal tubule dysfunction. In ambulatory settings, higher $\mathrm{Ua1m}$ is associated with acute kidney injury (AKI), progressive chronic kidney disease (CKD), cardiovascular (CV) events, and mortality. We investigated the associations of pre- and postoperative Ua1m concentrations with adverse outcomes after cardiac surgery. Methods: In 1,464 adults undergoing cardiac surgery in the prospective multicenter Translational Research Investigating Biomarker Endpoints for Acute Kidney Injury (TRIBE-AKI) cohort, we measured the pre-and postoperative Ua1m concentrations and calculated the changes from pre- to postoperative concentrations. Outcomes were postoperative AKI during index hospitalization and longitudinal risks for CKD incidence and progression, CV events, and all-cause mortality after discharge. We analyzed Ua1m continuously and categorically by tertiles using multivariable logistic regression and Cox
\end{abstract}

proportional hazards regression adjusted for demographics, surgery characteristics, comorbidities, baseline estimated glomerular filtration rate, urine albumin, and urine creatinine. Results: There were $230 \mathrm{AKI}$ events during cardiac surgery hospitalization; during median 6.7 years of follow-up, there were 212 cases of incident CKD, 54 cases of CKD progression, $269 \mathrm{CV}$ events, and 459 deaths. Each 2-fold higher concentration of preoperative $\mathrm{Ua} 1 \mathrm{~m}$ was independently associated with AKI (adjusted odds ratio [aOR] = 1.36, 95\% confidence interval 1.14-1.62), CKD progression (adjusted hazard ratio $[\mathrm{aHR}]=1.46,1.04-2.05)$, and all-cause mortality (aHR $=1.19,1.06-1.33)$ but not with incident CKD (aHR = $1.21,0.96-1.51$ ) or CV events ( $a H R=1.01,0.86-1.19$ ). Postoperative $\mathrm{Ua} 1 \mathrm{~m}$ was not associated with AKI (aOR per 2-fold higher $=1.07,0.93-1.22)$, CKD incidence $(\mathrm{aHR}=0.90,0.79$ 1.03 ) or progression (aHR $=0.79,0.56-1.11)$, CV events (aHR $=1.06,0.94-1.19)$, and mortality $(\mathrm{aHR}=1.01,0.92-1.11)$. Conclusion: Preoperative Ua $1 \mathrm{~m}$ concentrations may identify patients at high risk of AKI and other adverse events after cardiac surgery, but postoperative Ua1m concentrations do not appear to be informative.

(c) 2021 The Author(s).

Published by S. Karger AG, Basel karger@karger.com www.karger.com/ajn

Karger $\stackrel{\text { ' }}{5}$

BOPEN ACCESS
(C) 2021 The Author(s)

Published by S. Karger AG, Basel

This is an Open Access article licensed under the Creative Commons Attribution-NonCommercial-4.0 International License (CC BY-NC) (http://www.karger.com/Services/OpenAccessLicense), applicable to the online version of the article only. Usage and distribution for commercial purposes requires written permission.
Correspondence to:

Michael G. Shlipak, michael.shlipak@ucsf.edu 


\section{Introduction}

Kidney tubules are vital for fluid and electrolyte balance, neurohormonal regulation, and mineral metabolism, and kidney tubule injury can lead to severe kidney dysfunction and chronic kidney disease (CKD) $[1,2]$. However, current clinical practice lacks tools to monitor kidney tubule health directly, and serum creatinine remains the dominant biomarker for the assessment of kidney function and diagnosis of kidney injury. Creatinine reflects glomerular clearance but not kidney tubule injury or dysfunction, making it ill-suited for timely recognition of many forms of acute kidney injury (AKI), particularly those due to kidney tubule damage [3-5]. Because AKI is responsible for substantial morbidity and mortality and also portends important long-term outcomes, including risk of subsequent CKD $[6,7]$, cardiovascular (CV) disease [8-10], and mortality [11, 12], there is an impetus to identify biomarkers of kidney tubule injury and dysfunction that could improve AKI risk assessment and early diagnosis. Furthermore, several of these kidney tubule health biomarkers have been shown to predict long-term kidney, CV, and mortality outcomes in multiple populations and may add an important dimension to the assessment of kidney health [13-17].

One promising biomarker is alpha-1-microglobulin $(\alpha 1 \mathrm{~m})$, a low molecular weight protein synthesized by hepatocytes, freely filtered across the glomerulus, and then avidly reabsorbed by the proximal tubule $[18,19]$. Because the concentration of urine a $1 \mathrm{~m}(\mathrm{Ua} 1 \mathrm{~m})$ is inversely proportional to proximal tubule resorptive capacity, it can signal proximal tubule dysfunction: higher Ualm concentrations indicate impaired tubular reabsorption [20]. Small studies have previously shown that Ualm concentrations increase in AKI, reflect severity of AKI, and are associated with the need for dialysis after nonoliguric AKI [21-23]. Elevated Ua1m correlates with interstitial fibrosis and tubular atrophy on kidney biopsy, representing chronic kidney damage [24]. Furthermore, Ualm appears to predict kidney function decline and CKD progression [25, 26], AKI risk [27], CV events [28], and mortality $[16,28,29]$.

To better define its diagnostic and prognostic utility, we investigated $\mathrm{Ua} 1 \mathrm{~m}$ in a multicenter prospective cohort of adults at high risk for AKI who underwent cardiac surgery as part of the Translational Research Investigating Biomarker Endpoints for Acute Kidney Injury (TRIBE-AKI) study. Specifically, we measured preoperative and postoperative Ualm concentrations and evaluated their associations with postoperative AKI, CKD in- cidence and progression, CV events, and all-cause mortality after discharge. We hypothesized that higher pre- and postoperative levels of Ualm would be associated with adverse outcomes after cardiac surgery.

\section{Methods}

Study Design and Participants

TRIBE-AKI is a prospective, multicenter cohort study of adults who underwent cardiac surgery at 6 North American academic medical centers. Overall study design and cohort selection have been presented in detail previously [13, 14, 30]. Between July 2007 and December 2010, 1,601 adults at high risk for AKI who were undergoing coronary artery bypass graft (CABG) and/or valve surgery were enrolled after informed consent. Risk of AKI was determined by the presence of one of the following characteristics: emergency surgery, preoperative serum creatinine $>2.0 \mathrm{mg} / \mathrm{dL}$, ejection fraction $<35 \%$ or grade 3 or 4 left ventricular dysfunction, age $>70$ years, diabetes mellitus, concomitant CABG and valve surgery, or repeat revascularization. Patients undergoing multiple surgeries were enrolled in the study only once. Society of Thoracic Surgeon definitions were used for collection of preoperative characteristics, surgical characteristics, and postoperative complications [31]. To establish baseline kidney function, all participants had to have a documented value for serum creatinine within 2 months before surgery. This study complied with the Declaration of Helsinki, was approved by the institutional review board at each site, and all participants provided written informed consent.

\section{Sample Collection and Biomarker Measurement}

Sample collection and processing methods have been previously described in detail $[13,14]$. Briefly, urine and blood samples were collected preoperatively and then daily for up to 5 days after surgery. The postoperative samples used in this analysis were collected during the first $12 \mathrm{~h}$ of postoperative day 1 . All postoperative urine samples were collected before any form of dialysis was initiated. Fresh urine samples were obtained from the urimeter from participants with Foley catheters. Urine samples were centrifuged to separate cellular debris. Urine supernatant and plasma were stored at $-80^{\circ} \mathrm{C}$, until thawed for biomarker measurement. Ualm was measured at the Kidney Health Research Collaborative laboratory based at the San Francisco VA Health Care System (San Francisco, CA) by immunonephelometry on the Siemens BNII instrument using the proprietary $\mathrm{N}$ a1Microglobulin Reagent Kit (Siemens Medical Solutions USA, Inc., Malvern, PA) with a lower limit of detection (LLD) at $5.4625 \mathrm{mg} / \mathrm{L}$. Intra-assay coefficients of variation were not calculated because samples were run in a singlicate. A control sample provided by the manufacturer was run before batches of measurements to ensure validity of the assay. Values below the LLD were imputed as $5.4625 \mathrm{mg} / \mathrm{L}$ and included in the lowest tertile for categorical analyses.

\section{Outcomes}

Outcomes were defined and ascertained as they had been from the previous studies in the TRIBE-AKI cohort [30, 32-34]. AKI during index hospitalization was categorized as "at least mild AKI" defined as a serum creatinine increase $\geq 50 \%$ or $0.3 \mathrm{mg} / \mathrm{dL}$ from the preoperative level, and "severe AKI" defined as at least doubling of serum creatinine or need for dialysis during the index hospitalization [35]. 
Table 1. Baseline characteristics of the study population overall and by tertile of preoperative Ua $1 \mathrm{~m}$

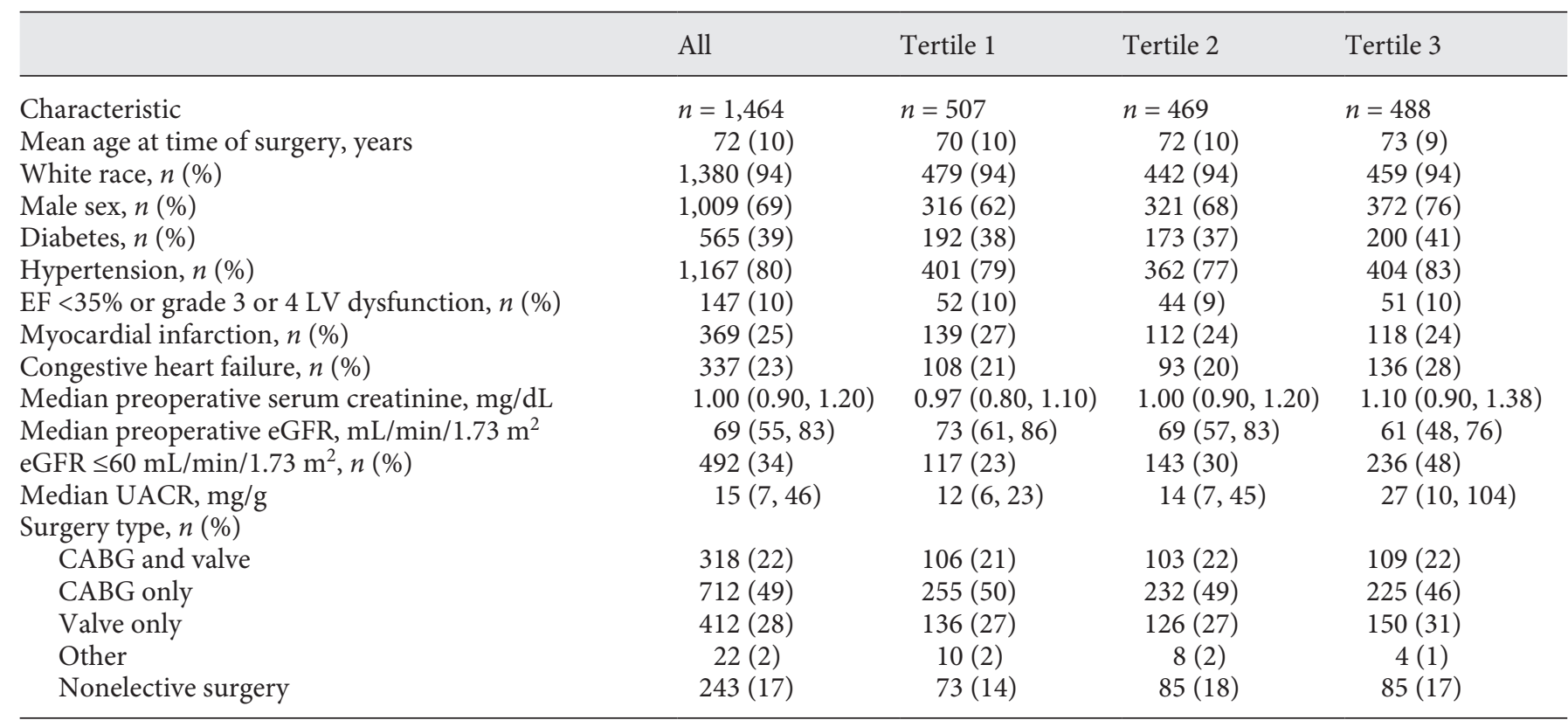

Ua1m ranges, tertile 1: 0-5.46 mg/L; tertile 2: 5.47-10.61 mg/L; and tertile 3: 10.63-285.02 mg/L. Values reported as mean (SD), $n$ (\%) or median (IQR). EF, ejection fraction; LV, left ventricular; eGFR, estimated glomerular filtration rate; UACR, urine albumin-tocreatinine ratio; $\mathrm{CABG}$, coronary artery bypass graft; $\mathrm{U} \alpha 1 \mathrm{~m}$, urine alpha-1-microglobulin; IQR, interquartile range.

Long-term outcomes among individuals who survived the index hospitalization were ascertained through patient phone calls, hospital records review (US sites), National Death Index search (US sites), and ICES data (Canadian site). CV events were defined as major adverse CV events: hospitalization for acute coronary syndrome, myocardial infarction, congestive heart failure, coronary bypass, and percutaneous coronary intervention. We identified CV events using administrative codes [36]. For US participants, CV events were obtained through linkages with Centers for Medicare and Medicaid Services databases. For Canadian participants, information was obtained from data held at ICES, linked using unique encoded identifiers, and analyzed at ICES.

$\mathrm{CKD}$ incidence and progression outcomes were available in participants from 2 study sites (Ontario, Yale). Follow-up serum creatinine values were obtained using the Ontario Laboratories Information System for Canadian participants and the Yale Joint Data Analytics Team's HELIX data repository for participants enrolled at Yale. Estimated glomerular filtration rate (eGFR) was calculated using the CKD Epidemiology Collaboration Equation [37]. For participants with preoperative eGFR $\geq 60 \mathrm{~mL} / \mathrm{min} / 1.73 \mathrm{~m}^{2}$, CKD incidence was defined as a $25 \%$ reduction in eGFR and a fall below $60 \mathrm{~mL} / \mathrm{min} / 1.73 \mathrm{~m}^{2}$. In participants with preoperative eGFR $<60 \mathrm{~mL} / \mathrm{min} / 1.73 \mathrm{~m}^{2}$, CKD progression was defined as a $50 \%$ reduction in eGFR or a fall below $15 \mathrm{~mL} / \mathrm{min} / 1.73 \mathrm{~m}^{2}[30,38,39]$.

\section{Statistical Analyses}

Descriptive statistics were reported using mean (standard deviation, $\mathrm{SD}$ ) or median (interquartile range [IQR]) for continuous variables and frequency (percentage) for categorical variables. We compared continuous variables using the Wilcoxon rank-sum test and categorical variables using the $\chi^{2}$ test. Ua1m was modeled continuously (after $\log _{2}$-transformation to represent per 2-fold higher levels) and categorically as tertiles. Logistic regression models were used to explore associations of (1) preoperative Ualm, (2) postoperative Ualm, and (3) relative change from pre-to postoperative Ualm levels with postoperative AKI. These models were adjusted for age, sex, race, preoperative comorbidities abstracted from chart review (diabetes, hypertension, heart failure, and myocardial infarction), baseline eGFR, urine albumin, and urine creatinine at corresponding time point, cardiopulmonary bypass time $>120$ min (omitted from models of preoperative Ualm), nonelective surgery, CABG versus valve replacement, and study site.

We used Cox proportional hazards regression to model the associations of (1) preoperative Ualm, (2) postoperative Ualm, and (3) relative change from pre- to postoperative Ualm levels with longitudinal outcomes after discharge, including CKD incidence, CKD progression, CV events, and all-cause mortality. Kolmogorov-type supremum tests were used to evaluate proportional hazards assumptions for all models. Models of CKD incidence and progression after discharge were adjusted for the variables listed above in the AKI model except for the addition of AKI or dialysis during index hospitalization and the exclusion of myocardial infarction history and surgery type (CABG vs. valve replacement). Models for CV events and all-cause mortality were also adjusted for similar variables as noted in the AKI model except for the addition of baseline smoking history, baseline BMI, and AKI or dialysis during index hospitalization, the exclusion of surgery type. 
Table 2. Association of preoperative Ua1m levels and risk of inhospital AKI and longitudinal adverse outcomes following hospital discharge in the TRIBE-AKI cohort

\begin{tabular}{|c|c|c|c|c|c|}
\hline & $\begin{array}{l}\mathrm{AKI}^{\mathrm{a}} \\
(n=1,464)\end{array}$ & $\begin{array}{l}\text { All-cause mortality } \\
(n=1,449)\end{array}$ & $\begin{array}{l}\text { CKD incidence }^{c} \\
(n=602)\end{array}$ & $\begin{array}{l}\text { CKD progression }{ }^{c} \\
(n=262)\end{array}$ & $\begin{array}{l}\text { CV events }{ }^{\mathrm{b}} \\
(n=1,449)\end{array}$ \\
\hline Events, $n(\%)$ & $230(16)$ & $459(32)$ & $212(35)$ & $54(21)$ & $269(19)$ \\
\hline Preoperative Ua1m & $\mathrm{aOR}(95 \% \mathrm{CI})$ & aHR (95\% CI) & aHR (95\% CI) & aHR (95\% CI) & aHR (95\% CI) \\
\hline Per 2-fold higher & $1.34(1.13,1.60)$ & $1.18(1.06,1.33)$ & $1.20(0.96,1.50)$ & $1.46(1.04,2.04)$ & $1.02(0.87,1.19)$ \\
\hline Tertile $2(5.47-10.61 \mathrm{mg} / \mathrm{L})$ & $0.64(0.43,0.95)$ & $0.93(0.71,1.22)$ & $1.36(0.98,1.88)$ & $0.72(0.30,1.74)$ & $0.83(0.60,1.14)$ \\
\hline Tertile $3(10.63-285.02 \mathrm{mg} / \mathrm{L})$ & $1.14(0.76,1.69)$ & $1.40(1.06,1.83)$ & $1.30(0.85,2.00)$ & $1.47(0.64,3.37)$ & $1.02(0.73,1.44)$ \\
\hline
\end{tabular}

Ua1m ranges, tertile 1:0-5.46 mg/L; tertile 2: 5.47-10.61 mg/L; and tertile 3: 10.63-285.02 mg/L. Ua1m, urine alpha-1-microglobulin; AKI, acute kidney injury; TRIBE-AKI, Translational Research Investigating Biomarker Endpoints for Acute Kidney Injury; CKD,

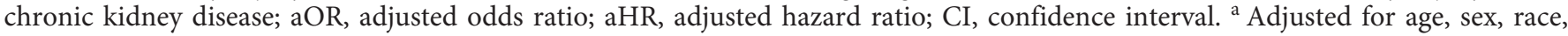
nonelective surgery, CABG versus valve replacement, diabetes, hypertension, congestive heart failure, myocardial infarction, baseline eGFR, urine albumin, urine creatinine, and site. ${ }^{\mathrm{b}}$ Adjusted for age, sex, race, nonelective surgery, diabetes, hypertension, congestive heart failure, myocardial infarction, smoking, BMI, AKI/dialysis during index hospitalization, baseline eGFR, urine albumin, urine creatinine, and site. ${ }^{c}$ Adjusted for age, sex, race, nonelective surgery, diabetes, hypertension, congestive heart failure, AKI/dialysis during index hospitalization, baseline eGFR, urine albumin, urine creatinine, and site.

All tests of statistical significance were two-sided, and $p<0.05$ was considered statistically significant. To investigate effect modification by heart failure, we tested for interactions between heart failure status with CV events and all-cause mortality. Analyses were performed in SAS (version 9.4; SAS Institute, Cary, NC, USA).

\section{Results}

The size of the analytic cohort differed by outcome (Tables 2-4). In the 1,464 participants with preoperative Ualm measurements, mean age was 72 years, $69 \%$ were male, and $94 \%$ were White (Table 1). Surgery was nonelective in $17 \%$ and consisted of CABG alone in $49 \%$ of participants. The preoperative Ualm level was below LLD in nearly $35 \%$ of participants. By tertile of preoperative $\mathrm{Ua} 1 \mathrm{~m}$, there were 507 participants in tertile 1 (all of whom had levels below LLD), 469 participants in tertile 2 , and 488 participants in tertile 3 . In the highest Ua $1 \mathrm{~m}$ tertile compared to the lowest, participants were older (73 vs. 70 years), more likely to be male (76 vs. $62 \%$ ), and had a higher prevalence of CKD, diabetes, hypertension, and heart failure.

\section{Preoperative Ua1m Levels and Postoperative \\ Outcomes}

There were 230 AKI events during the index hospitalization, among which 67 were stage 2 or 3 . Analyzed con- tinuously, each 2-fold higher level of Ualm was associated with significantly increased odds of postoperative AKI (Table 2; Fig. 1). In the categorical analysis, the association between the highest tertile of preoperative Ua $1 \mathrm{~m}$ and postoperative AKI was attenuated and not statistically significant. In stratified analysis by diabetes status, there was evidence that Ualm was more strongly associated with postoperative AKI among participants without diabetes compared to those with diabetes (online suppl. Table 1; for all online suppl. material, see www. karger.com/doi/10.1159/00010.1159/000518240). In unadjusted analyses, there were statistically significant associations between preoperative $\mathrm{Ua} 1 \mathrm{~m}$ and stage 2 or 3 AKI (unadjusted odds ratio $[\mathrm{OR}]=1.36,95 \%$ confidence interval [CI] 1.09-1.69), but these were no longer significant in the adjusted model (adjusted OR $=1.30,95 \%$ CI $0.97-1.73$ ).

There were 459 deaths over a median follow-up of 6.7 years (IQR 4.0, 7.9). Higher preoperative Ualm levels were associated with increased risk of all-cause mortality when analyzed continuously and categorically (Table 2; Fig. 2). The highest tertile of Ualm was associated with a $40 \%$ higher risk of all-cause mortality compared to the lowest tertile. However, this association was modified by heart failure (Ualm and heart failure interaction $p=0.009$ for continuous analysis, $p=0.008$ for categorical analysis). Among those without heart failure, preoperative Ualm was strongly associated with mortality 
Table 3. Association of postoperative Ua1m levels and risk of inhospital AKI and longitudinal adverse outcomes following hospital discharge in the TRIBE-AKI cohort

\begin{tabular}{|c|c|c|c|c|c|}
\hline & $\begin{array}{l}\mathrm{AKI}^{\mathrm{a}} \\
(n=1,445)\end{array}$ & $\begin{array}{l}\text { All-cause mortality } \\
(n=1,430)\end{array}$ & $\begin{array}{l}\text { CKD incidence }{ }^{c} \\
(n=598)\end{array}$ & $\begin{array}{l}\text { CKD progression } \\
(n=258)\end{array}$ & $\begin{array}{l}\text { CV events }{ }^{\mathrm{b}} \\
(n=1,430)\end{array}$ \\
\hline Events, $n(\%)$ & $226(16)$ & $450(31)$ & $210(35)$ & $53(21)$ & $262(18)$ \\
\hline Postoperative Ualm & $\mathrm{aOR}(95 \% \mathrm{CI})$ & aHR $(95 \% \mathrm{CI})$ & aHR (95\% CI) & aHR (95\% CI) & aHR (95\% CI) \\
\hline Per 2-fold higher & $1.07(0.93,1.22)$ & $1.01(0.92,1.11)$ & $0.90(0.79,1.03)$ & $0.79(0.56,1.11)$ & $1.06(0.94,1.19)$ \\
\hline Tertile $1(5.46-8.05 \mathrm{mg} / \mathrm{L})$ & 1.00 (reference) & 1.00 (reference) & 1.00 (reference) & 1.00 (reference) & 1.00 (reference) \\
\hline Tertile $2(8.06-23.03 \mathrm{mg} / \mathrm{L})$ & $0.75(0.47,1.17)$ & $0.97(0.73,1.30)$ & $1.02(0.66,1.57)$ & $2.16(0.93,5.05)$ & $1.55(1.05,2.27)$ \\
\hline Tertile $3(23.20-651.36 \mathrm{mg} / \mathrm{L})$ & $0.98(0.61,1.58)$ & $1.14(0.83,1.59)$ & $0.84(0.52,1.38)$ & $0.74(0.22,2.45)$ & $1.56(1.01,2.39)$ \\
\hline
\end{tabular}

Postoperative samples collected on postoperative day 1. U $1 \mathrm{~m}$, urine alpha-1-microglobulin; AKI, acute kidney injury; TRIBE-AKI, Translational Research Investigating Biomarker Endpoints for Acute Kidney Injury; CKD, chronic kidney disease; aOR, adjusted odds ratio; aHR, adjusted hazard ratio; $\mathrm{CI}$, confidence interval. ${ }^{a}$ Adjusted for age, sex, race, cardiopulmonary bypass time $>120$ min, nonelective surgery, CABG versus valve replacement, diabetes, hypertension, congestive heart failure, myocardial infarction, baseline eGFR, urine albumin, urine creatinine, and site. ${ }^{b}$ Adjusted for age, sex, race, cardiopulmonary bypass time $>120$ min, nonelective surgery, diabetes, hypertension, congestive heart failure, myocardial infarction, smoking, BMI, AKI/dialysis during index hospitalization, baseline eGFR, urine albumin, urine creatinine, and site. ${ }^{c}$ Adjusted for age, sex, race, cardiopulmonary bypass time $>120$ min, nonelective surgery, diabetes, hypertension, congestive heart failure, AKI/dialysis during index hospitalization, baseline eGFR, urine albumin, urine creatinine, and site.

Table 4. Association of per $100 \%$ change in preoperative to postoperative day $1 \mathrm{Ua} 1 \mathrm{~m}$ levels and risk of inhospital AKI and longitudinal adverse outcomes following hospital discharge in the TRIBE-AKI cohort

\begin{tabular}{|c|c|c|c|c|c|}
\hline & $\begin{array}{l}\mathrm{AKI}^{\mathrm{a}} \\
(n=1,444)\end{array}$ & $\begin{array}{l}\text { All-cause mortality } \\
(n=1,429)\end{array}$ & $\begin{array}{l}\text { CKD incidence }{ }^{c} \\
(n=598)\end{array}$ & $\begin{array}{l}\text { CKD progression } \\
(n=258)\end{array}$ & $\begin{array}{l}\text { CV events }{ }^{\mathrm{b}} \\
(n=1,429)\end{array}$ \\
\hline Events, $n(\%)$ & $226(16)$ & $450(31)$ & $210(35)$ & $53(21)$ & $262(18)$ \\
\hline Change in Ualm & $\mathrm{aOR}(95 \% \mathrm{CI})$ & aHR (95\% CI) & aHR (95\% CI) & aHR (95\% CI) & aHR (95\% CI) \\
\hline per $100 \%$ change & $1.00(0.97,1.02)$ & $0.98(0.96,1.00)$ & $0.98(0.95,1.01)$ & $0.79(0.65,0.97)$ & $0.99(0.97,1.02)$ \\
\hline Tertile $2(0.0-152.9 \%)$ & $0.74(0.49,1.11)$ & $0.83(0.65,1.06)$ & $0.62(0.42,0.94)$ & $0.55(0.26,1.17)$ & $1.02(0.73,1.43)$ \\
\hline Tertile 3 (154.1-840.7\%) & $0.95(0.61,1.47)$ & $0.78(0.58,1.04)$ & $0.69(0.43,1.09)$ & $0.32(0.11,0.92)$ & $1.03(0.70,1.50)$ \\
\hline
\end{tabular}

U $\alpha 1 \mathrm{~m}$, urine alpha-1-microglobulin; AKI, acute kidney injury; TRIBE-AKI, Translational Research Investigating Biomarker Endpoints for Acute Kidney Injury; CKD, chronic kidney disease; aOR, adjusted odds ratio; aHR, adjusted hazard ratio; CI, confidence interval. a Adjusted for age, sex, race, cardiopulmonary bypass time $>120 \mathrm{~min}$, nonelective surgery, CABG versus valve replacement, diabetes, hypertension, congestive heart failure, myocardial infarction, baseline eGFR, urine albumin, urine creatinine, and site. ${ }^{\mathrm{b}}$ Adjusted for age, sex, race, cardiopulmonary bypass time $>120 \mathrm{~min}$, nonelective surgery, diabetes, hypertension, congestive heart failure, myocardial infarction, smoking, BMI, AKI/dialysis during index hospitalization, baseline eGFR, urine albumin, urine creatinine, and site. ${ }^{\mathrm{c}}$ Adjusted for age, sex, race, cardiopulmonary bypass time $>120 \mathrm{~min}$, nonelective surgery, diabetes, hypertension, congestive heart failure, AKI/dialysis during index hospitalization, baseline eGFR, urine albumin, urine creatinine, and site.

(adjusted hazard ratio $[\mathrm{aHR}]=1.28,95 \%$ CI 1.12-1.47), whereas the association was weaker among those with heart failure ( $\mathrm{aHR}=1.06,95 \%$ CI 0.84-1.32). Categorical analyses yielded similar findings (online suppl. Table 2).

Urine Alpha-1-Microglobulin and Adverse Outcomes after Cardiac Surgery
There were 212 incident CKD diagnoses and 54 events of CKD progression over a median follow-up of 5.8 years (IQR 4.2, 7.1 years). There were no significant associations between preoperative Ualm and CKD incidence (Table 2). However, there was a significant in- 


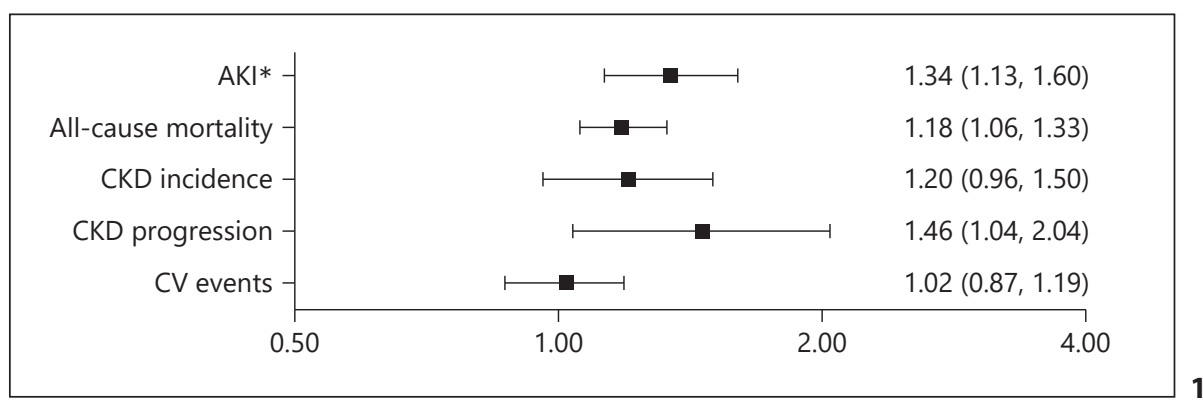

Fig. 1. Adjusted associations of preoperative Ualm levels with risk of in-hospital AKI and longitudinal adverse outcomes following hospital discharge in the TRIBEAKI cohort. Squares represent model-adjusted estimates of OR (for AKI) or HR (longitudinal outcomes) for each 2-fold higher preoperative U $\alpha 1 \mathrm{~m}$ concentration. Brackets represent 95\% CIs. *For AKI, measure of association is aOR. OR, odds ratio; aOR, adjusted odds ratio; $\mathrm{U} \alpha 1 \mathrm{~m}$, urine alpha-1-microglobulin; AKI, acute kidney injury; TRIBE-AKI, Translational Research Investigating Biomarker Endpoints for Acute Kidney Injury; CI, confidence interval; HR, hazard ratio.

Fig. 2. Kaplan-Meier curves depicting survival probability by tertile of preoperative Ualm. Ualm, urine alpha-1-microglobulin.

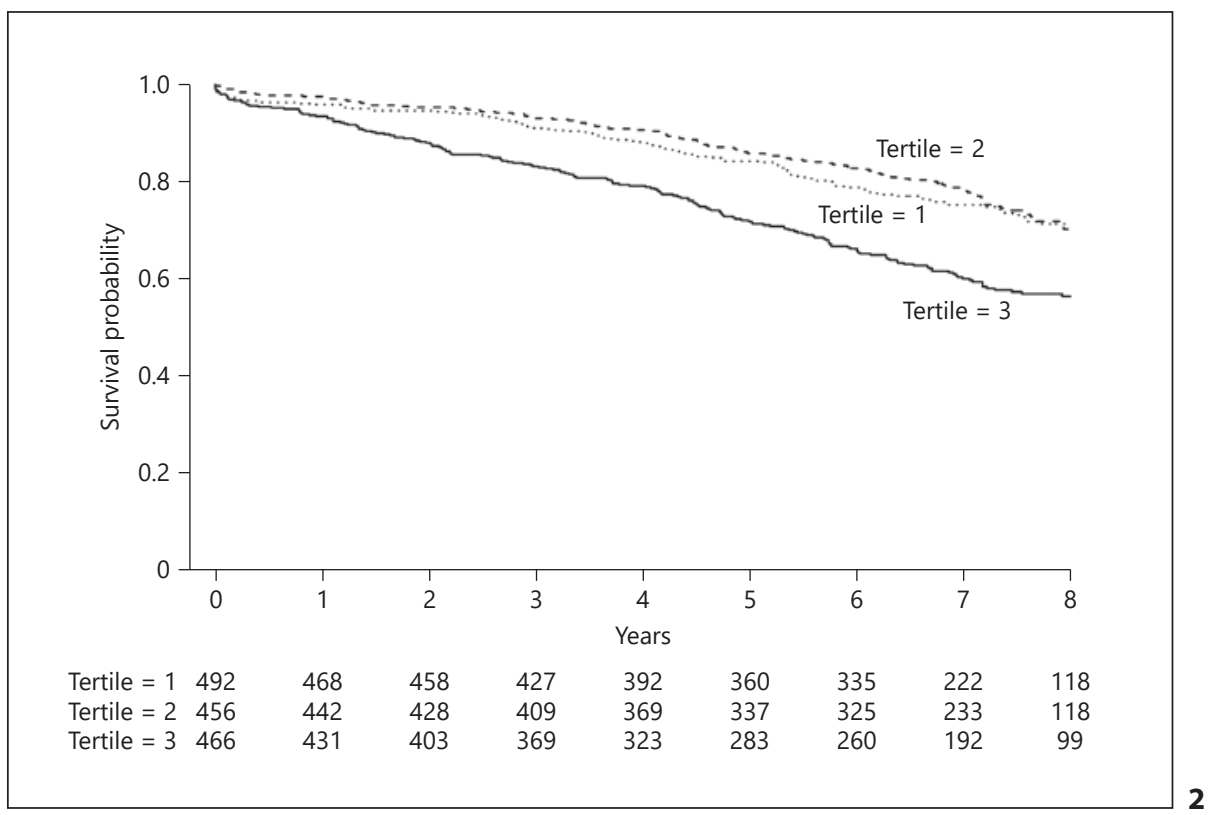

teraction on diabetes status, whereby preoperative Ualm was associated with incident CKD among participants with diabetes $(\mathrm{aHR}=1.73,95 \%$ CI $1.30-2.31$ ) but not among those without diabetes $(\mathrm{aHR}=0.87,95 \%$ CI 0.61-1.25; online suppl. Table 3). Higher preoperative Ualm concentrations were associated with significantly greater risk of CKD progression when analyzed as a continuous predictor, though this association was not statistically significant when Ua $1 \mathrm{~m}$ was analyzed in tertiles (Table 2; Fig. 1).

There were $269 \mathrm{CV}$ events over a median follow-up of 6.3 years (IQR 3.1, 7.7). Preoperative Ua1m levels were not associated with CV events in continuous or categorical analyses.

\section{Postoperative Day 1 Ua1m Levels and Postoperative Outcomes}

In the unadjusted analyses, postoperative day $1 \mathrm{Ua} 1 \mathrm{~m}$ levels were associated with higher odds of AKI (OR =
$1.28,95 \%$ CI 1.16-1.41) and severe AKI (OR $=1.50,95 \%$ CI 1.27-1.76), but these associations were attenuated and no longer significant after multivariable adjustment (Table 3; online suppl. Table 1). Postoperative Ua1m was not associated with CKD incidence or CKD progression. In contrast, higher postoperative Ualm concentrations were associated with increased risk of CV events. Though the strength of this association was attenuated by multivariable adjustment, the categorical estimates remained significant and directionally consistent in the multivariable model (Table 3). Heart failure status did not modify the association of postoperative Ualm with CV events. Postoperative Ualm levels were not associated with allcause mortality (Table 3 ).

\section{Relative Changes from Preoperative to Postoperative}

Ualm Levels and Postoperative Outcomes

The median (IQR) change from preoperative Ualm level to postoperative day $1 \mathrm{Ua} 1 \mathrm{~m}$ level was $43.9 \%$ 
Fig. 3. a Frequency distribution of preoperative Ualm (red line) and postoperative Ualm (blue line) concentrations in $\mathrm{mg} / \mathrm{L}$. U $\alpha 1 \mathrm{~m}$, urine alpha-1-microglobulin. b Histogram of relative (\%) change from preoperative to postoperative Ualm concentration. Ua1m, urine alpha-1-microglobulin.

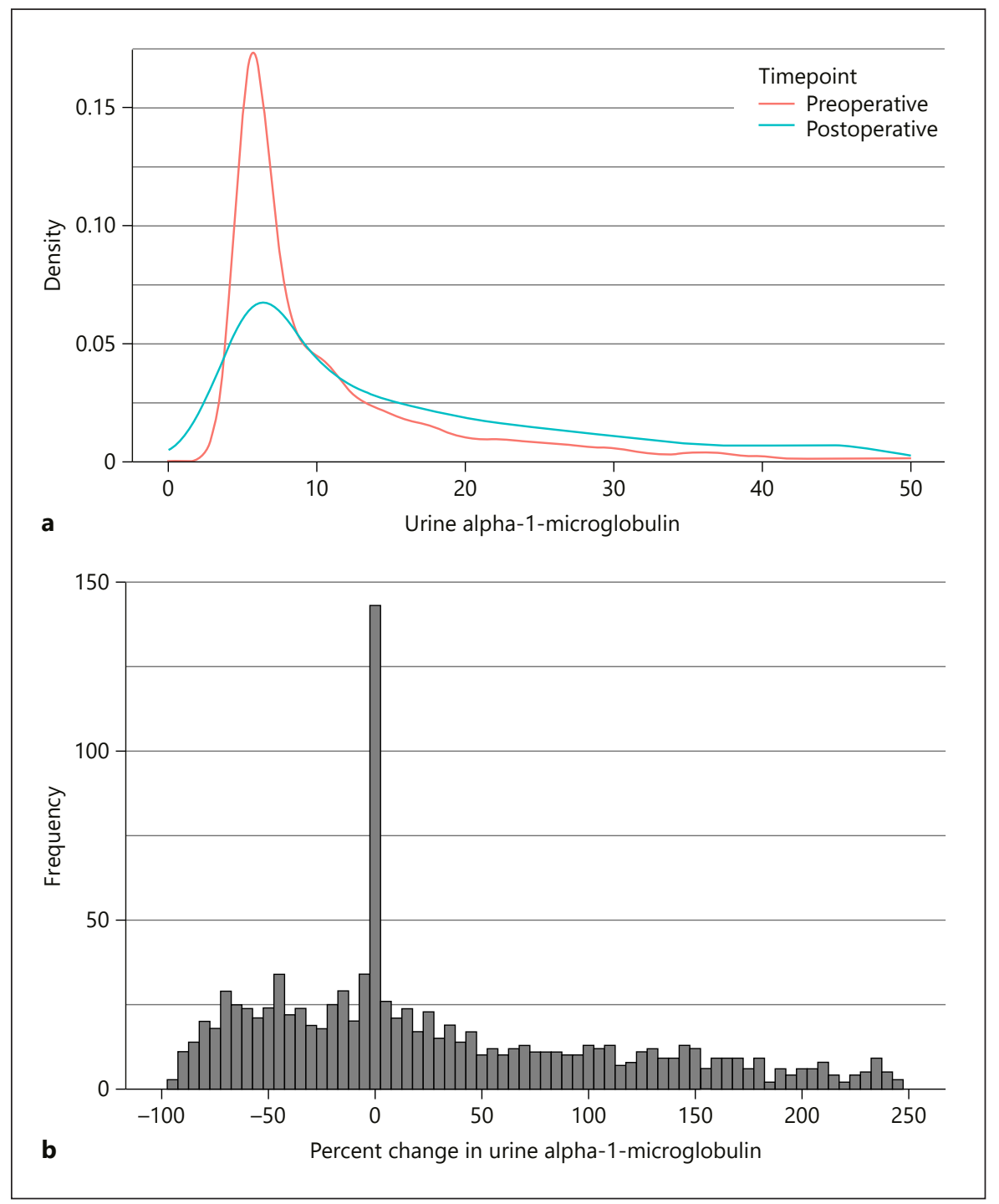

$(-12.2 \%, 256.9 \%)$ (Fig. 3a, b). Larger increases from preoperative to postoperative day 1 levels were associated with marginally increased risk of AKI, but these associations disappeared with adjustment (Table 4). Larger increases in Ualm were not associated with CKD incidence but were associated with lower risk of CKD progression (for each $100 \%$ increase in Ualm aHR $=0.79,95 \%$ CI 0.65-0.97). Compared to the lowest tertile, the highest tertile of change in Ualm levels was also associated with decreased risk of CKD progression $(\mathrm{aHR}=0.32,95 \% \mathrm{CI}$ 0.11-0.92; Table 4). There were no significant associations between the percent change of $\mathrm{Ua} 1 \mathrm{~m}$ and $\mathrm{CV}$ events. Associations between changes in Ualm and all-cause mortality were marginal and attenuated with adjustment (Table 4).

Urine Alpha-1-Microglobulin and Adverse Outcomes after Cardiac Surgery

\section{Discussion}

In this large, well-described cohort of high-risk patients undergoing cardiac surgery, we found that higher preoperative Ualm levels were significantly associated with increased risk of AKI during index hospitalization as well as with CKD progression and all-cause mortality. Postoperative Ua1m levels and the relative change between pre- and postoperative Ualm were not strongly associated with post-discharge outcomes. Our findings underscore the potential value of $\mathrm{Ua} 1 \mathrm{~m}$ as an ambulatory marker of tubular function that can risk-stratify as well as prognosticate kidney and nonkidney outcomes but did not support the use of $\mathrm{Ualm}$ as an early indicator of inhospital AKI. 
Ua1m levels reflect proximal tubule dysfunction and have been reported to increase in AKI and to correlate with pathologic changes of CKD, making Ualm an attractive surrogate for kidney damage $[18,22,24]$. However, the finding that preoperative Ua1m was more consistently and strongly associated with AKI, CKD, and death compared with postoperative Ualm was unexpected. Perhaps due to the unique kinetics of $\alpha 1 \mathrm{~m}$, preoperative (baseline) and immediate postoperative (stressed) levels appear to represent distinct physiologies and should be interpreted differently based on our findings. Because $\alpha 1 \mathrm{~m}$ is filtered across the glomerulus, it is susceptible to hemodynamic variation during and after cardiac surgery and may be unreliable in the early postoperative period compared to the steady state preoperative levels. Though postoperative levels of Ualm increased in most participants, some of these elevations may have quickly declined as normal tubular function was restored, but we measured Ualm only on the first postoperative day and thus could not observe normalization. Transient elevations Ualm might not capture clinically important AKI in contrast to the tubule injury markers that we have previously studied in this cohort, such as IL-18 and KIM-1, in which the median levels rise approximately 10-fold in AKI cases compared to noncases [13, 40-42]. In contrast, Ua1m may be most informative of underlying kidney health when measured in the absence of acute stressors.

Our finding that higher preoperative Ualm was associated with $36 \%$ higher odds of postoperative AKI is consistent with previously published estimates. Among ambulatory SPRINT trial participants with CKD, each 2 -fold higher baseline Ua1m level was associated with $20 \%$ higher risk of subsequent inhospital AKI, independent of baseline eGFR, albuminuria, and other kidney tubule biomarkers [27]. Likewise, higher Ualm appears to portend worsening kidney function. We found that higher baseline Ualm levels indicated a significantly greater risk of CKD progression in participants with prevalent CKD and demonstrated a directionally consistent, though nonsignificant, association with incident CKD. These results agree with findings from ambulatory settings in which Ua $1 \mathrm{~m}$ predicted faster kidney function decline in healthy and chronically ill populations $[25,26]$. Furthermore, we found some evidence that the association between preoperative Ualm levels and kidney outcomes might differ depending on baseline diabetes status, which warrants investigation in future studies. In sum, the results of our analysis, in addition to those from prior studies, suggest that baseline Ualm levels might indicate the kidney's sus- ceptibility to insults and risk of functional decline independent of baseline eGFR and albuminuria.

Unexpectedly, larger Ualm elevations in the immediate postoperative period were associated with significantly lower risk of CKD progression among participants with prevalent CKD. However, we did not find significant associations between postoperative $\mathrm{Ua} 1 \mathrm{~m}$ levels alone and AKI or CKD incidence; rather, this association was observed when we analyzed the relative changes in the Ua $1 \mathrm{~m}$ level from before to after surgery. Few studies have evaluated associations of changes in Ualm with subsequent CKD risk. One recent study in ambulatory HIV-infected women observed that rising Ualm concentrations were associated with higher risk of incident CKD [43]. Additionally, among SPRINT trial participants assigned to the usual care arm, rising Ualm concentrations were associated with incident $\mathrm{CKD}$, whereas declining Ua1m concentrations were associated with incident CKD in the intensive antihypertensive therapy arm [44]. These findings suggest that changes in Ualm may disclose risk of CKD, but also that Ualm concentrations are susceptible to hemodynamic changes. This issue may be particularly relevant to the period immediately after cardiac surgery. The degree of perioperative change may be further confounded by underlying kidney health, as demonstrated by a study comparing Ua $1 \mathrm{~m}$ levels before and after cardiac surgery between individuals with creatinine clearances above and below $60 \mathrm{~mL} / \mathrm{min}$ [21]. In this study, the relative change from preoperative to postoperative levels was greater among those with creatinine clearance $>60 \mathrm{~mL} / \mathrm{min}$ [21]. Thus, it is conceivable that larger relative increases in Ualm levels after surgery are an indicator of individuals with healthier kidneys before surgery. Acute variations in Ualm warrant further exploration to clarify their potential significance.

Higher preoperative Ualm levels in this cohort were independently associated with all-cause mortality, which is consistent with findings across a variety of populations. In kidney transplant recipients, Ua $1 \mathrm{~m}$ was associated with a $51 \%$ increased risk of death per 2-fold higher level [45]. Among 2,948 Framingham Heart Study participants, each standard deviation increase of log-transformed Ualm was associated with $26 \%$ higher risk of all-cause mortality [29]. In a casecohort study of ambulatory older adults, each 2 -fold higher baseline Ualm was associated with a 1.29-fold greater risk of all-cause mortality over a median follow-up of 12 years [16]. Similarly, Garimella et al. [28] demonstrated that each 2 -fold higher Ua1m level was 
associated with a $25 \%$ increase in the adjusted hazard of all-cause mortality among patients with CKD and hypertension.

The association of Ualm with mortality risk in this study was much stronger in participants without heart failure than in those with heart failure. The physiology of Ualm may render it less reliable in patients with heart failure. Heart failure can cause neurohormonally mediated reductions in GFR [46], which could decrease the amount of $\alpha 1 \mathrm{~m}$ filtered into the tubule and ultimately excreted in urine irrespective of reabsorption - in essence, mimicking intact reabsorption. However, this was unlikely to be the case in the TRIBE-AKI cohort, given that participants with baseline heart failure were more likely to be in the highest tertile of preoperative Ualm. Alternatively, mortality risk in individuals with heart failure may be influenced by pathways that are less related to kidney tubule dysfunction [36].

Previously reported associations between $\mathrm{Ua} 1 \mathrm{~m}$ and heart disease have been inconsistent, and we found only weak evidence for an association between Ualm and $\mathrm{CV}$ events in this cohort. Similarly, the aforementioned analysis from the Framingham Heart Study found no association between Ualm and CV events despite a strong association with mortality [29]. Jotwani et al. [16] found that higher baseline Ualm was associated with significantly higher risk of CV events but not incident heart failure in older adults. Similarly, Park et al. [45] found that higher Ua1m was associated with CV events in kidney transplant recipients. Among TRIBEAKI participants, those with severe postoperative AKI were more likely to experience $\mathrm{CV}$ events or death during a median 3.8 years of follow-up; however, only postoperative cardiac biomarkers - not kidney injury biomarkers - were associated with this outcome, suggesting against an independent kidney pathway in this relationship [36].

Though this study benefited from a well-characterized, large, multicenter cohort, its observational design is susceptible to residual confounding despite comprehensive multivariable adjustment. TRIBE-AKI did not record information on the severity and control of some comorbidities that could conceivably obfuscate the association between Ualm and postoperative outcomes. However, we presume that comorbidities were perioperatively optimized for elective surgeries, which comprised the majority $(83 \%)$ of procedures. Furthermore, only $10 \%$ of participants had severe heart failure at baseline as defined by left ventricular ejection fraction $<35 \%$ or grade 3 or 4 left ventricular dysfunction. The unique clinical characteristics of the participants limit generalizability, particularly to populations with lower burdens of CVD and lower risk of AKI. Furthermore, the sample was overwhelmingly White and male, highlighting the need for future studies to include participants more representative of the general population. Postoperative Ualm levels were available only from the first postoperative day, so it is unknown whether or not Ualm fluctuations later in the hospitalization would be informative for kidney and CV prognosis. In spite of these limitations, the validity of our findings is supported by their consistency with results from studies of Ualm in other populations.

In conclusion, preoperative Ualm was independently associated with postoperative AKI, CKD progression, and all-cause mortality in this cohort of adults undergoing cardiac surgery. Contrary to our a priori hypothesis, associations of postoperative $\mathrm{Ua} 1 \mathrm{~m}$ or changes in pre- to postoperative Ualm concentrations with these outcomes were weak and inconsistent. These results contribute to growing evidence that Ualm levels have important associations with adverse outcomes and suggest that kidney tubule dysfunction is an important risk factor for kidney disease and mortality. Future studies should better characterize short-, medium-, and long-term variations in $\mathrm{Ua} 1 \mathrm{~m}$ levels and investigate whether these associations generalize to more diverse populations.

\section{Acknowledgements}

This study was supported by ICES, which is funded by an annual grant from the Ontario Ministry of Health and Long-Term Care (MOHLTC). The opinions, results, and conclusions reported in this article are those of the authors and are independent from the funding sources. No endorsement by ICES or the Ontario MOHLTC is intended or should be inferred. Parts of this material are based on data and/or information compiled and provided by CIHI. However, the analyses, conclusions, opinions, and statements expressed in the material are those of the authors and not necessarily those of CIHI.

\section{Statement of Ethics}

This study was performed in accordance with the World Medical Association Declaration of Helsinki. The study protocol was approved by the institutional review board at each study site. All study participants provided written informed consent.
Urine Alpha-1-Microglobulin and

Adverse Outcomes after Cardiac Surgery
Am J Nephrol 2021;52:673-683

DOI: $10.1159 / 000518240$ 


\section{Conflict of Interest Statement}

C.R.P. serves on the advisory boards of Renalytix AI, LLC, and GENFIT Pharmaceuticals. M.M.E. and M.G.S. have received funding from Bayer Healthcare Pharmaceuticals, Inc. M.G.S. discloses consulting income from Intercept Pharmaceuticals, Inc. and Cricket Health. The remaining authors did not declare relevant financial interests.

\section{Funding Sources}

J.G.A. is supported by National Institute of Diabetes and Digestive and Kidney Diseases of the National Institutes of Health under Award No. F32DK126381. CRP is supported by NIH Grants under Award No. R01HL085757, U01DK082185, and the P30DK079310 O’Brien Kidney Center Grant.

\section{Author Contributions}

J.G.A., M.M.E., C.R.P., and M.G.S. contributed to research question and study design; C.R.P., A.X.G., and M.G.S. contributed to data acquisition; J.G.A., M.M.E., A.X.G., S.G.C., H.T.P., E.M., C.R.P., and M.G.S. contributed to data analysis and interpretation; H.T.P. and E.M. contributed to statistical analysis; M.M.E., C.R.P., and M.G.S. contributed to supervision and mentorship. Each author contributed to important intellectual content during manuscript drafting and revision.

\section{Data Availability Statement}

The data that support the findings of this study are not publicly available due to their containing information that could compromise the privacy of research participants but are available from the corresponding author (M.G.S.) upon reasonable request.

\section{References}

1 Liu BC, Tang TT, Lv LL, Lan HY. Renal tubule injury: a driving force toward chronic kidney disease. Kidney Int. 2018 Mar;93(3):568-79.

2 Yu AS, Chertow GM, Luyckx VA, Marsden PA, Skorecki K, Taal MW, editors. Brenner \& Rector's the kidney. 11th ed. Philadelphia, PA: Elsevier; 2020.

3 Rule AD, Amer H, Cornell LD, Taler SJ, Cosio FG, Kremers WK, et al. The association between age and nephrosclerosis on renal biopsy among healthy adults. Ann Intern Med. 2010 May 4;152(9):561-7.

4 Thiele RH, Isbell JM, Rosner MH. AKI associated with cardiac surgery. Clin J Am Soc Nephrol. 2015 Mar 6;10(3):500-14.

5 Molitoris BA. Beyond biomarkers: machine learning in diagnosing acute kidney injury. Mayo Clin Proc. 2019 Mar;94(5):748-50.

6 He L, Wei Q, Liu J, Yi M, Liu Y, Liu H, et al. AKI on CKD: heightened injury, suppressed repair, and the underlying mechanisms. Kidney Int. 2017 Nov;92(5):1071-83.

7 Levey AS, James MT. Acute kidney injury. Ann Intern Med. 2017 Nov 7;167(9):ITC66-80.

8 Sarnak MJ, Levey AS, Schoolwerth AC, Coresh J, Culleton B, Hamm LL, et al. Kidney disease as a risk factor for development of cardiovascular disease: a statement from the American Heart Association Councils on Kidney in Cardiovascular Disease, High Blood Pressure Research, Clinical Cardiology, and Epidemiology and Prevention. Circulation. 2003 Nov;108(17):2154-69.

9 Coresh J, Astor B, Sarnak MJ. Evidence for increased cardiovascular disease risk in patients with chronic kidney disease. Curr Opin Nephrol Hypertens. 2004 Jan;13(1):73-81.

10 Odutayo A, Wong CX, Farkouh M, Altman DG, Hopewell S, Emdin CA, et al. AKI and long-term risk for cardiovascular events and mortality. J Am Soc Nephrol. 2017 Jan;28(1): $377-87$.
11 Go AS, Chertow GM, Fan D, McCulloch CE, Hsu CY. Chronic kidney disease and the risks of death, cardiovascular events, and hospitalization. N Engl J Med. 2004 Sep 23;351(13): 1296-305.

12 Coca SG, Yusuf B, Shlipak MG, Garg AX, Parikh CR. Long-term risk of mortality and other adverse outcomes after acute kidney injury: a systematic review and meta-analysis. Am J Kidney Dis. 2009 Jun;53(6):961-73.

13 Parikh CR, Coca SG, Thiessen-Philbrook H, Shlipak MG, Koyner JL, Wang Z, et al. Postoperative biomarkers predict acute kidney injury and poor outcomes after adult cardiac surgery. J Am Soc Nephrol. 2011 Sep;22(9): 1748-57.

14 Parikh CR, Devarajan P, Zappitelli M, Sint K, Thiessen-Philbrook H, Li S, et al. Postoperative biomarkers predict acute kidney injury and poor outcomes after pediatric cardiac surgery. J Am Soc Nephrol. 2011 Sep;22(9): 1737-47.

15 Peralta CA, Katz R, Bonventre JV, Sabbisetti V, Siscovick D, Sarnak M, et al. Associations of urinary levels of kidney injury molecule 1 (KIM-1) and neutrophil gelatinase-associated lipocalin (NGAL) with kidney function decline in the multi-ethnic study of atherosclerosis (MESA). Am J Kidney Dis. 2012 Dec; 60(6):904-11.

16 Jotwani V, Katz R, Ix JH, Gutiérrez OM, Bennett M, Parikh CR, et al. Urinary biomarkers of kidney tubular damage and risk of cardiovascular disease and mortality in elders. Am J Kidney Dis. 2018 Aug;72(2):205-13.

17 Malhotra R, Craven T, Ambrosius WT, Killeen AA, Haley WE, Cheung AK, et al. Effects of intensive blood pressure lowering on kidney tubule injury in CKD: a longitudinal subgroup analysis in SPRINT. Am J Kidney Dis. 2019 Jan; 73(1):21-30.
18 Itoh Y, Kawai T. Human alpha 1-microglobulin: its measurement and clinical significance. J Clin Lab Anal. 1990;4(5):376-84.

19 Weber MH, Verwiebe R. Alpha 1-microglobulin (protein HC): features of a promising indicator of proximal tubular dysfunction. Eur J Clin Chem Clin Biochem. 1992 Oct;30(10):683-91.

20 Penders J, Delanghe JR. Alpha 1-microglobulin: clinical laboratory aspects and applications. Clin Chim Acta. 2004 Aug 16;346(2): 107-18.

21 Dehne MG, Boldt J, Heise D, Sablotzki A, Hempelmann G. [Tamm-Horsfall protein, alpha-1- and beta-2-microglobulin as kidney function markers in heart surgery]. Anaesthesist. 1995 Aug;44(8):545-51.

22 Edelstein CL, Faubel S. Biomarkers in acute kidney injury. Biomarkers in kidney disease. Amsterdam: Elsevier Press; 2011. p. 177-232.

23 Heise D, Rentsch K, Braeuer A, Friedrich M, Quintel M. Comparison of urinary neutrophil glucosaminidase-associated lipocalin, cystatin $\mathrm{C}$, and a1-microglobulin for early detection of acute renal injury after cardiac surgery. Eur J Cardiothorac Surg. 2011 Jan;39(1):38-43.

24 Amer H, Lieske JC, Rule AD, Kremers WK, Larson TS, Franco Palacios CR, et al. Urine high and low molecular weight proteins oneyear post-kidney transplant: relationship to histology and graft survival. Am J Transplant. 2013 Mar;13(3):676-84.

25 Jotwani V, Scherzer R, Abraham A, Estrella MM, Bennett M, Cohen MH, et al. Association of urine a1-microglobulin with kidney function decline and mortality in HIV-infected women. Clin J Am Soc Nephrol. 2015 Jan 7;10(1):63-73.

26 Ascher SB, Scherzer R, Estrella MM, Shlipak MG, Ng DK, Palella FJ, et al. Associations of urine biomarkers with kidney function decline in HIV-infected and uninfected men. Am J Nephrol. 2019 Sep 25;50:401-10. 
27 Bullen AL, Katz R, Lee AK, Anderson CAM, Cheung AK, Garimella PS, et al. The SPRINT trial suggests that markers of tubule cell function in the urine associate with risk of subsequent acute kidney injury while injury markers elevate after the injury. Kidney Int. 2019 May;96(2):470-9.

28 Garimella PS, Lee AK, Ambrosius WT, Bhatt U, Cheung AK, Chonchol M, et al. Markers of kidney tubule function and risk of cardiovascular disease events and mortality in the SPRINT trial. Eur Heart J. 2019 Jul 30;40(42): 3486-93.

29 O'Seaghdha CM, Hwang SJ, Larson MG, Meigs JB, Vasan RS, Fox CS. Analysis of a urinary biomarker panel for incident kidney disease and clinical outcomes. J Am Soc Nephrol. 2013 Nov;24(11):1880-8.

30 Menez S, Moledina DG, Garg AX, ThiessenPhilbrook H, McArthur E, Jia Y, et al. Results from the TRIBE-AKI Study found associations between post-operative blood biomarkers and risk of chronic kidney disease after cardiac surgery. Kidney Int. 2020 Jul 25;99(3): 716-24.

31 Fernandez FG, Falcoz PE, Kozower BD, Salati M, Wright CD, Brunelli A. The society of thoracic surgeons and the European society of thoracic surgeons general thoracic surgery databases: joint standardization of variable definitions and terminology. Ann Thorac Surg. 2015 Jan;99(1):368-76.

32 Coca SG, Garg AX, Thiessen-Philbrook H, Koyner JL, Patel UD, Krumholz HM, et al. Urinary biomarkers of AKI and mortality 3 years after cardiac surgery. J Am Soc Nephrol. 2014 May;25(5):1063-71.
33 Mansour SG, Zhang WR, Moledina DG, Coca SG, Jia Y, Thiessen-Philbrook H, et al. The association of angiogenesis markers with acute kidney injury and mortality after cardiac surgery. Am J Kidney Dis. 2019 Jul;74(1):36-46.

34 Patel DM, Thiessen-Philbrook H, Brown JR, McArthur E, Moledina DG, Mansour SG, et al. Association of plasma-soluble ST2 and galectin-3 with cardiovascular events and mortality following cardiac surgery. Am Heart J. 2020 Feb;220:253-63.

35 Mehta RL, Kellum JA, Shah SV, Molitoris BA, Ronco C, Warnock DG, et al. Acute kidney injury network: report of an initiative to improve outcomes in acute kidney injury. Crit Care. 2007;11:R31.

36 Parikh CR, Puthumana J, Shlipak MG, Koyner JL, Thiessen-Philbrook H, McArthur E, et al. Relationship of kidney injury biomarkers with long-term cardiovascular outcomes after cardiac surgery. J Am Soc Nephrol. 2017 Dec; 28(12):3699-707.

37 Levey AS, Stevens LA, Schmid CH, Zhang YL, Castro AF 3rd, Feldman HI, et al. A new equation to estimate glomerular filtration rate. Ann Intern Med. 2009;150(9):604-12.

38 Go AS, Parikh CR, Ikizler TA, Coca S, Siew ED, Chinchilli VM, et al. The assessment, serial evaluation, and subsequent sequelae of acute kidney injury (ASSESS-AKI) study: design and methods. BMC Nephrol. 2010 Aug 27;11:22.

39 Hsu CY, Chinchilli VM, Coca S, Devarajan P, Ghahramani N, Go AS, et al. Post-acute kidney injury proteinuria and subsequent kidney disease progression: the Assessment, Serial Evaluation, and Subsequent Sequelae in Acute Kidney Injury (ASSESS-AKI) Study. JAMA Intern Med. 2020 Jan 27;180(3):40210.
40 Koyner JL, Garg AX, Coca SG, Sint K, Thiessen-Philbrook H, Patel UD, et al. Biomarkers predict progression of acute kidney injury after cardiac surgery. J Am Soc Nephrol. 2012 May;23(5):905-14.

41 Parikh CR, Thiessen-Philbrook H, Garg AX, Kadiyala D, Shlipak MG, Koyner JL, et al. Performance of kidney injury molecule- 1 and liver fatty acid-binding protein and combined biomarkers of AKI after cardiac surgery. Clin J Am Soc Nephrol. 2013 Jul;8(7):1079-88.

42 Coca SG, Nadkarni GN, Garg AX, Koyner J, Thiessen-Philbrook H, McArthur E, et al. First Post-operative urinary kidney injury biomarkers and association with the duration of AKI in the TRIBE-AKI cohort. PLoS One. 2016;11(8): 0161098.

43 Ascher SB, Scherzer R, Estrella MM, Muiru AN, Jotwani VK, Grunfeld C, et al. Kidney tubule health scores and their associations with incident CKD in women living with HIV. HIV Med. 2021 Mar 9;22(7):527-37.

44 Zhang WR, Craven TE, Malhotra R, Cheung AK, Chonchol M, Drawz P, et al. Kidney damage biomarkers and incident chronic kidney disease during blood pressure reduction: a case-control study. Ann Intern Med. 2018 Nov 6;169(9):610-8.

45 Park M, Katz R, Shlipak MG, Weiner D, Tracy $\mathrm{R}$, Jotwani $\mathrm{V}$, et al. Urinary markers of fibrosis and risk of cardiovascular events and death in kidney transplant recipients: the FAVORIT trial. Am J Transplant. 2017 Oct; 17(10):2640-9.

46 Verbrugge FH, Guazzi M, Testani JM, Borlaug BA. Altered hemodynamics and end-organ damage in heart failure: impact on the lung and kidney. Circulation. 2020 Sep 8; 142(10):998-1012. 